\title{
Transposition
}

Musique et Sciences Sociales

$7 \mid 2018$

Le prix de la musique

Frédéric Ramel \& Cécile Prévost-Thomas (eds), International Relations, Music and Diplomacy, Sounds and Voices on the International Stage

cham, Palgrave Macmillan, 2018.

\section{Stéphanie Gonçalves}

\section{CpenEdition}

\section{Journals}

Édition électronique

URL : http://journals.openedition.org/transposition/2257

DOI : $10.4000 /$ transposition.2257

ISSN : $2110-6134$

Éditeur

CRAL - Centre de recherche sur les arts et le langage

Référence électronique

Stéphanie Gonçalves, «Frédéric Ramel \& Cécile Prévost-Thomas (eds), International Relations, Music and Diplomacy, Sounds and Voices on the International Stage », Transposition [En ligne], 7| 2018, mis en ligne le 15 septembre 2018, consulté le 25 septembre 2020. URL : http://journals.openedition.org/ transposition/2257 ; DOI : https://doi.org/10.4000/transposition.2257

Ce document a été généré automatiquement le 25 septembre 2020

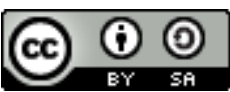

La revue Transposition est mise à disposition selon les termes de la Licence Creative Commons Attribution - Partage dans les Mêmes Conditions 4.0 International. 


\title{
Frédéric Ramel \& Cécile Prévost- Thomas (eds), International Relations, Music and Diplomacy, Sounds and Voices on the International Stage
}

Cham, Palgrave Macmillan, 2018.

\author{
Stéphanie Gonçalves
}

\section{RÉFÉRENCE}

Frédéric Ramel \& Cécile Prévost-Thomas (ed.), International Relations, Music and

Diplomacy, Sounds and Voices on the International Stage, Cham, Palgrave Macmillan, 2018.

1 Comment comprendre et analyser la diplomatie musicale dans une perspective pluridisciplinaire, internationale et diachronique ? Publié en février 2018, l'ouvrage collectif International Relations, Music and Diplomacy, Sounds and Voices on the International Stage, dirigé par Frédéric Ramel et Cécile Prévost-Thomas, répond à ce défi. Frédéric Ramel est directeur du département de Science Politique à Sciences-Po Paris, spécialiste, entre autres, des liens entre art et politique dans une dimension internationale. Cécile Prévost-Thomas est maîtresse de conférences à la Sorbonne Nouvelle - Paris 3 en sociologie de la musique, spécialiste des médiations musicales et de la chanson française en particulier. Ce livre est le fruit d'une conférence internationale organisée en avril 2016 à Paris $^{1}$, dans laquelle les disciplines représentées sont diverses: des chercheurs en histoire, en sociologie, en musicologie ou en science politique jettent un regard pluriel sur la place et les rôles de la musique dans la diplomatie internationale.

2 La publication s'inscrit dans le développement récent et particulièrement fécond de l'étude de la musique dans la diplomatie internationale - au même titre que celui du théâtre ou de la danse depuis plusieurs années - développement qui a eu lieu 
principalement dans la recherche anglophone ${ }^{2}$. Après les tournants "acoustique " (p.2) et «culturel» (p.3) qui ont permis de croiser musicologie et relations internationales, l'étude de la diplomatie musicale se porte sur de nouveaux enjeux. Longtemps cantonnées à la question de la place de la musique dans les conflits notamment la période prolifique de la guerre froide - et aux seuls acteurs étatiques principalement les États-Unis - les recherches se développent aujourd'hui vers les acteurs non-étatiques et tendent à développer une vision globale. En France, elle reste encore un terrain en chantier, malgré les recherches dynamiques d'Anaïs Fléchet, d'Esteban Buch, d'Anne-Sylvie Barthel-Calvet ou de Fanny Gribenski, des contributeurs du livre. L'objectif, réussi, de l'ouvrage collectif est de mettre en avant tant la multiplicité des définitions de la «musique» - sons, voix et instruments - que la multiplicité des regards portés sur l'objet, grâce à l'utilisation de concepts communs, notamment celui de « scènes » du spécialiste de la communication Will Straw ${ }^{3}$.

3 L'ouvrage, composé de dix chapitres, plus une introduction de C. Prévost-Thomas et $\mathrm{F}$. Ramel et un article de synthèse final de Jessica Gienow-Hecht, la spécialiste berlinoise de la discipline, revient sur les liens complexes entre musique et diplomatie. L'ouvrage est divisé en trois parties, (1) Shaping the Musical Scenes. Sounds and Voices as Objectives of Diplomacy, (2) Shaping the Diplomatic Scene. Sounds and Voices as Frameworks of Diplomacy, (3) Bringing Music to the Fore of the Diplomatic Scene. Sounds and voices as Objects of Diplomacy, qui dressent un panorama international de la place des sons sur les " scènes » diplomatiques, y compris dans ses coulisses. L'introduction, particulièrement pertinente, resitue les enjeux de la diplomatie musicale et l'état de l'art qui y est fait sera fort utile aux chercheurs qui découvrent la diplomatie musicale dans une perspective diachronique et multidisciplinaire. Géographiquement, les contributions embrassent trois continents - on regrettera l'absence de l'Asie qui reste un terrain encore à défricher. Chronologiquement, elles vont du XvII (le chapitre 2 de Michela Berti sur la ville de Rome au cœur des échanges musicaux européens) au xxI siècle (le chapitre 10 de Dean Vuletic sur l'Eurovision) mais la plupart se concentrent sur le $\mathrm{xx}^{\mathrm{e}}$ siècle, en particulier la guerre froide, une période déjà bien étudiée dans l'historiographie car les sources sont disponibles. À noter, l'index particulièrement détaillé comprenant noms de personnes, concepts, instruments ou institutions et, pour chaque article, notes et bibliographie dans lesquelles le chercheur curieux dénichera plus aisément des références.

4 Le premier intérêt de l'ouvrage réside dans la volonté des auteurs d'interroger les multiples facettes des "sons" diplomatiques, à travers des concepts transversaux. Celui de "scènes» de Will Straw, bien qu'il définisse la structure du livre en trois parties, n'est malheureusement pas développé par un certain nombre de contributions. Cependant, il est pertinent pour aller au-delà des concepts fréquemment utilisés en histoire culturelle ou en relations internationales comme ceux de représentations, de prestige ou de soft power. En dépassant la scène physique associée aux actions d'écoute, de performance et de contrôle (p. 5-6), les scènes « restreintes » ou « ouvertes » (p. 6) de Straw permettent de mettre en avant les dynamiques de circulations locales, nationales et internationales et de visibilité et d'invisibilité.

5 Le second intérêt est sans conteste l'article conclusif de Gienow-Hecht (p. 259) qui fait la synthèse des contributions et interroge chacune d'entre elles sous l'angle d'un concept opérant: celui de "nation branding». La difficulté, comme souvent avec les ouvrages collectifs, réside dans le fait de trouver des points communs et transversaux 
dans un grand nombre d'articles. Ce concept, développé il y a une quinzaine d'années et d'abord associé au marketing, permet de dépasser les conclusions, parfois ambigües, de chaque article et de parvenir de manière plus concrète aux résultats. Au-delà de la question désormais bien connue des acteurs étatiques et de leur contrôle sur la musique, le nation branding permet d'ouvrir la recherche vers les acteurs non-étatiques, de multiplier les études sur les outils acoustiques utilisés (par exemple, un hymne national ou des vidéos de promotion) et de questionner, de manière diachronique, les ruptures et les continuités de la diplomatie musicale dans le temps long. La chercheuse allemande dépasse les enjeux de prestige généralement attribués à la diplomatie musicale, ceux de " jeu et de projection ", pour interroger les "rêves et de désirs », qui disent encore plus sur la fabrique des sons dans la diplomatie.

L'ouvrage intéressera assurément tout autant les musicologues et les historiens culturels que les chercheurs en sociologie ou en relations internationales. En particulier, les chercheurs qui s'occupent de la diplomatie du sport, des échanges scientifiques et artistiques, notamment du théâtre ou de la danse, trouveront des références utiles et des concepts fructueux - comme celui de "scènes » ou de diplomatie « hybride» (A-S. Barthel-Calvet, p. 65) - qui nourriront leur recherche. La diplomatie musicale, et plus généralement les liens entre musique et politique, apparaissent comme des chantiers de recherche féconds, qui s'alimenteront encore de l'ouverture progressive d'archives. Concluons, avec Jessica Gienow-Hecht, en appelant de nos vœux à un développement d'une narration transnationale, transversale et transdisciplinaire des liens entre diplomatie et musique.

\section{NOTES}

1. «Sounds and Voices on the International Stage: Understanding Musical Diplomacies", Sciences Po Paris, conférence organisée par le CERI de Sciences Po et le CERLIS (Université Paris Descartes CNRS - Université Sorbonne Nouvelle), les 20 et 21 Avril 2016. Voir en ligne, http:// www.sciencespo.fr/ceri/fr/content/sounds-and-voices-international-stage-understandingmusical-diplomacies, consulté le 17 juin 2018.

2. Par exemple, AHREndT Rebecca, FerRAguto Mark et MAHIET Damien (eds), Music and Diplomacy from the Early Modern Era to the Present, New York, Palgrave Macmillan, 2014 ; GIENOW-HECHT Jessica (ed.), Music and International History in the Twentienth Century, New York/Oxford, Berghahn Books, 2015.

3. STRAW Will, "Scènes : ouvertes et restreintes ", Cahiers de recherche sociologique, $\mathrm{n}^{\circ}$ 57, 2014, p. 17-32. 


\section{AUTEURS}

\section{STÉPHANIE GONÇALVES}

Stéphanie Gonçalves est historienne, post-doctorante chargée de recherche au Fonds National de la Recherche Scientifique belge à l'Université libre de Bruxelles. Elle a soutenu en 2015 une thèse de doctorat portant sur la diplomatie culturelle du ballet pendant la Guerre froide (Danser pendant la guerre froide, 1945-1968, qui sortira aux Presses Universitaires de Rennes en septembre 2018). Elle a été assistante à l'ULB pendant six ans, post-doctorante à l'Academia Belgica de Rome et ATER à l'Université de Rennes 2. Elle est spécialiste du lien entre danse et politique au XXème siècle et s'intéresse en particulier aux circulations transnationales des danseurs, notamment les danseurs soviétiques. Son projet de recherches actuel s'intitule « Repenser le phénomène Béjart, perspectives socio-culturelles sur un chorégraphe multi-facette». 